\title{
« Il commanda qu'elle fust rasée et démolie jusques aux fondemens ". La destruction de la ville et de la cathédrale de Thérouanne (Pas-de-Calais) en 1553
}

Olivier Blamangin, Laetitia Dalmau et Jérôme Maniez

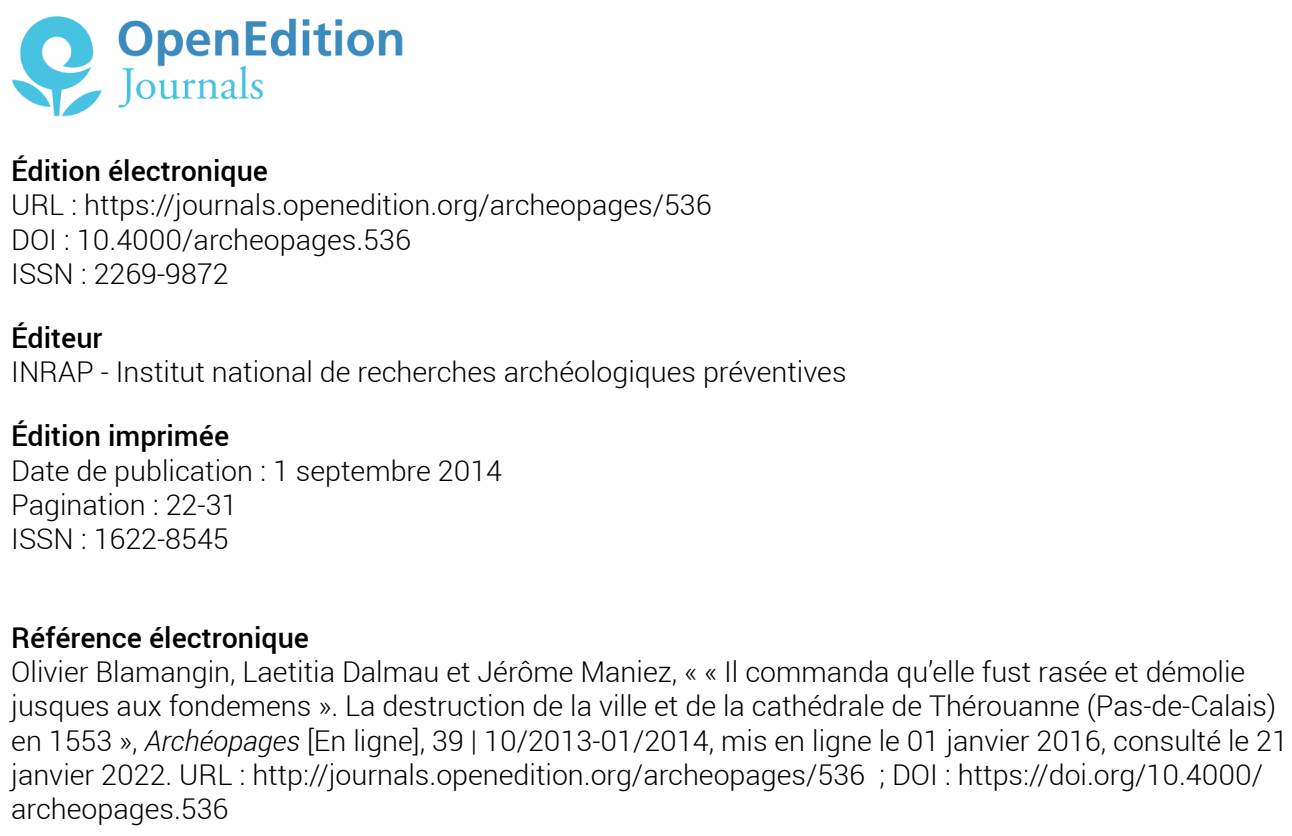




\section{« Il commanda qu'elle fust rasée et démolie jusques aux fondemens ${ }^{1}$ La destruction de la ville et de la cathédrale de Thérouanne (Pas-de-Calais) en 1553}

\author{
Olivier Blamangin Inrap, UMR 8164 Halma-Ipel \\ Laetitia Dalmau Conseil général du Pas-de-Calais \\ Jérôme Maniez Conseil général du Pas-de-Calais
}

\author{
«O noble cité jollie \\ Qui Morine eut à nom, \\ Tu estoit belle et jollie \\ Jadis et de grand regnon; \\ Et se les coups de canon \\ Te ont détruit par feu et flamme [...] » \\ (Complainte de Thérouene, éd. Quenson, 1855).
}

\section{2}

1. Ordre de Charles Quint selon Rabutin, 1932, p. 206.
Le 20 juin 1553, après sept semaines de siège, les troupes de Charles Quint prenaient la ville de Thérouanne, enclave française en comté d'Artois. L’empereur réserva à la cité épiscopale un châtiment exceptionnel : plutôt que de s'en tenir aux pillages d'usage, il ordonna la destruction totale de la ville, de ses fortifications et de ses édifices religieux. L'armée impériale s'attaqua ensuite à la place-forte voisine d'Hesdin, également possession de la couronne de France, qui tomba le 18 juillet avant d'être rasée à son tour. Thérouanne ne se relèvera jamais de ce démantèlement, tandis que la forteresse d'Hesdin sera finalement reconstruite, à quelques kilomètres de la ville détruite. La démolition de ces deux villes est sans équivalent pour l'époque. Comme le souligne Pieter Martens, il ne s'agit pas « d'une ruine ponctuelle provoquée par des troupes de passage mais, au contraire, d'une entreprise visant à leur éradication complète » (Martens, 2007, p. 66).

Ancien chef-lieu de la Civitas Morinorum, la Cité des Morins (sur Thérouanne antique, voir notamment Delmaire, 1976 ; Delmaire 1984 ; Blamangin et al., 2011), et siège d'un vaste diocèse depuis les premières décennies du VII ${ }^{\mathrm{e}}$ siècle (Mériaux, 2000), la ville de Thérouanne n'avait pas, au milieu du XVI ${ }^{\mathrm{e}}$ siècle, la prospérité économique ou le rayonnement spirituel de sa proche voisine, Saint-Omer, avec sa collégiale et son abbaye SaintBertin. Affectée par les sièges à répétition, isolée en territoire ennemi [ill. 1], la cité végétait au plan démographique (Guyotjeannin, 2010). Elle restait pourtant un lieu de pouvoir, siège d'un évêché aux revenus importants. La mise en évidence, à l'occasion de certaines interventions archéologiques [cf. encadré p.zz26], de travaux d'embellissement

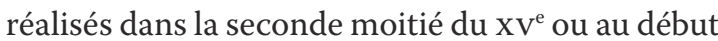
$\mathrm{du} \mathrm{XVI}^{\mathrm{e}}$ siècle pourrait témoigner d'une reprise économique tardive à la fin du Moyen Âge (Barret et al., 2005). Thérouanne était surtout, au milieu du XVI ${ }^{\mathrm{e}}$ siècle, une pièce maîtresse dans le dispositif militaire de la couronne de France sur sa frontière nord. François I ${ }^{\text {er }}$ avait coutume de dire qu'elle était « un des deux oreillers sur lesquels le roi de France peut dormir en paix ». En réalité, la position était fragile (Vissière, 200o) et, dans le conflit qui opposait alors la maison des Valois aux Habsbourg, la cité fut assiégée à six reprises et changea cinq fois de maître entre 1477 et 1553 (Delmaire, 1999). En 1513, les armées impériales et anglaises, qui s'étaient emparées de Thérouanne, s'empressèrent de démanteler ses défenses et détruisirent en grande partie la ville. Restituée à la couronne de France, la cité voit ses fortifications relevées dans les années 1515-1520 et modernisées après un nouveau siège en 1537, pour mieux résister aux assauts de l'artillerie (Martens, 2011). Finalement, le sort réservé à la ville en 1553 « ne fut pas un accident de l'histoire, mais un aboutissement » (Delmaire, 1999, p. 127).

Les chroniqueurs du $\mathrm{XVI}^{\mathrm{e}}$ siècle ont relaté en détail le déroulement du siège de 1553, mais les traces archéologiques des opérations militaires sont rares. Un important réseau fossoyé, mis au jour en 1995 à l'occasion d'une fouille sur le contournement de la ville (Blamangin, 1996), pourrait témoigner des travaux d'approche engagés par les pionniers. L'ensemble compte jusqu'à douze fossés parallèles creusés sur la ligne de crête d'une butte de sables landéniens, au nordouest et en surplomb de la batterie d'artillerie $\mathrm{du}$ « vieux château ». Ces structures, qui alternent des comblements anthropiques intervenant très rapidement après creusement et des comblements naturels par ruissellement, peuvent sans doute être interprétées comme les négatifs des palissades protégeant les tranchées des assiégeants. Le 


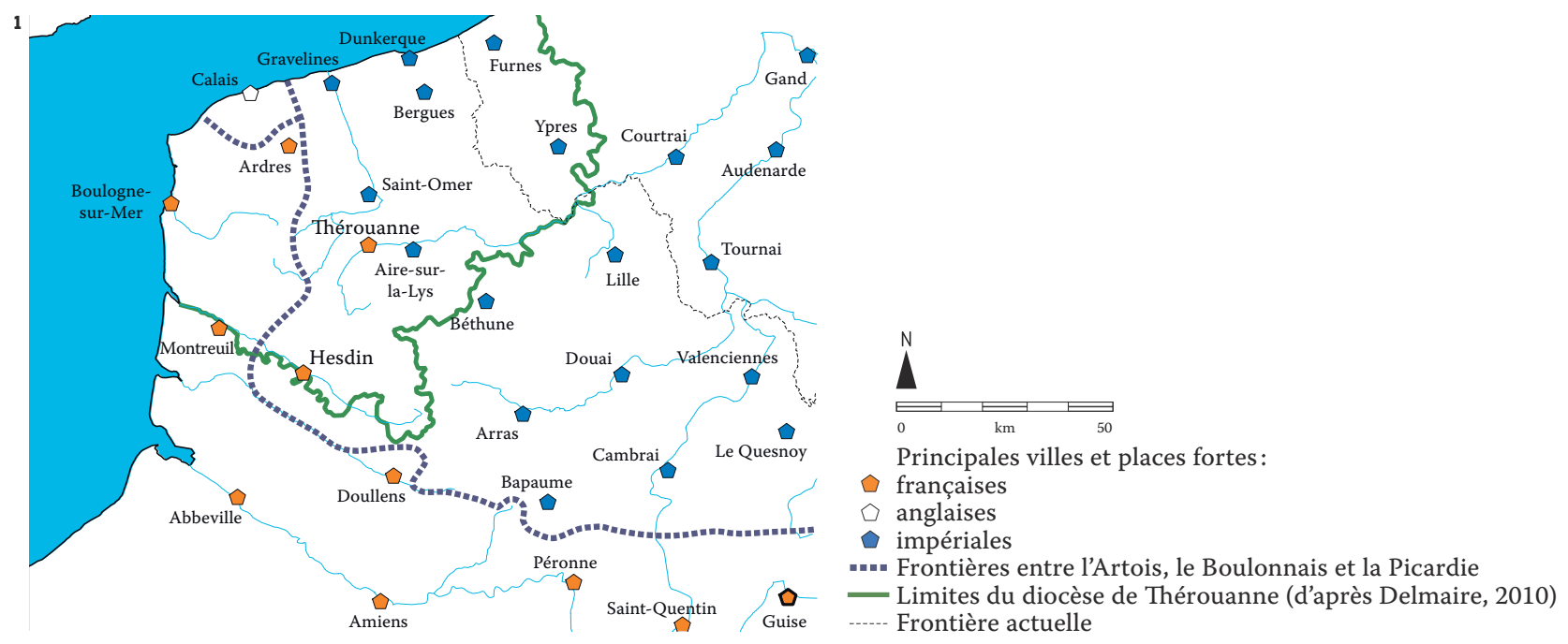

1. Thérouanne, place forte française isolée en terre impériale, était au milieu du xvie siècle le siège d'un évêché dont le ressort s'étendait de part et d'autre de la frontière, sur le Boulonnais et une partie de l'Artois et de la Picardie.

2. et 3. Les représentations du siège de Thérouanne en 1553 témoignent de

l'ampleur des destructions liées aux opérations militaires. On observe

ici une large brèche dans le mur d'enceinte ainsi que la ruine partielle de la tour de la cathédrale

Notre-Dame.

2. Le siège de Thérouanne, vue depuis le nord-ouest. Gravure sur bois de 1553 , conservée au British

Museum, coloriée à la main, imprimée sur quatre

feuilles par Heylrich Zeel à Strasbourg.

3. Siège de la ville

de Thérouanne en 1553.

Détail d'une gravure

du xviII ${ }^{\mathrm{e}}$ siècle, copie d'après un original disparu.
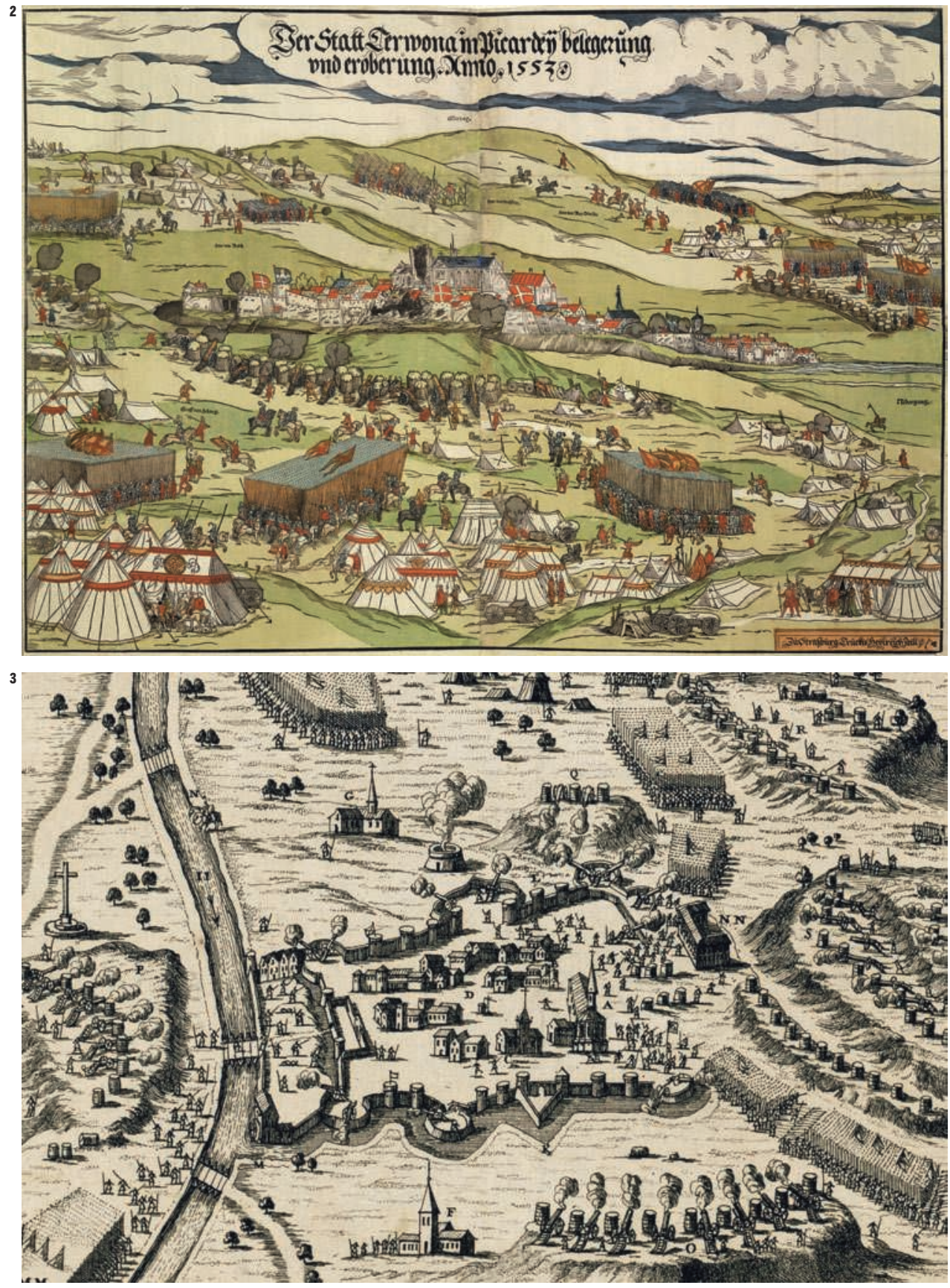


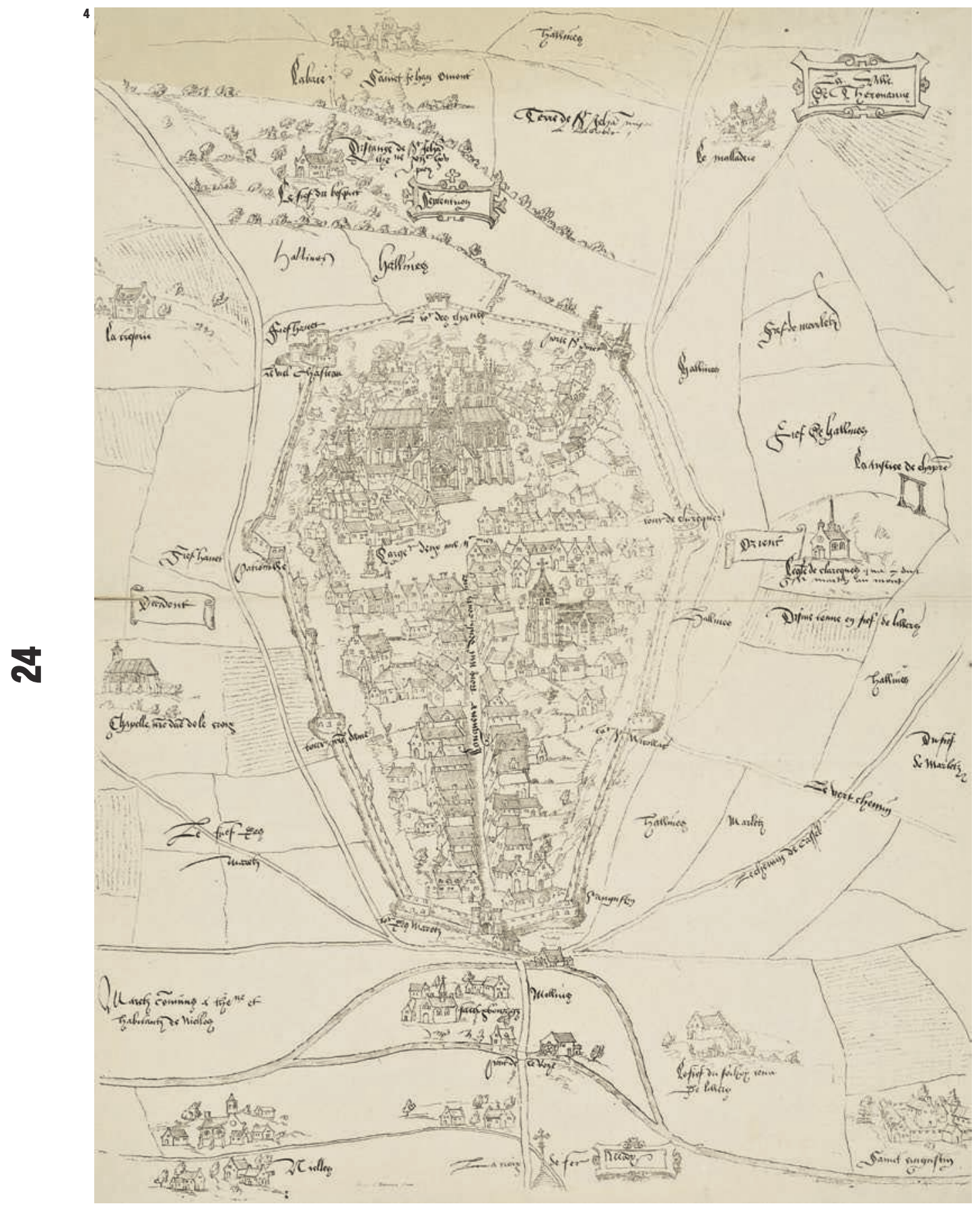

4. La vue cavalière

de 1539 figure, outre

la cathédrale de Thérouanne,

l'église Saint-Nicolas et,

extra-muros, l'église

du faubourg Saint-Esprit

(au sud), l'église Saint-

Martin-au-Mont (à l'est)

ainsi que l'abbaye

de Saint-Augustin

(au sud-est). Tous ces

édifices religieux furent

détruits en 1553.

L'abbaye Saint-Jean-au-

Mont (au nord) avait

été ruinée à l'occasion

d'un précédent siège.

$5 a$ et $b$. Les interventions

archéologiques mettent

au jour les vestiges

de l'habitat civil de

la ville de Thérouanne,

arasé suite à la prise

de la ville par les troupes

impériales.

5a. Diagnostic rue

Saint-Jean, parcelle AB

249, 2006.

5b. Diagnostic rue

Saint-Jean, parcelle AB
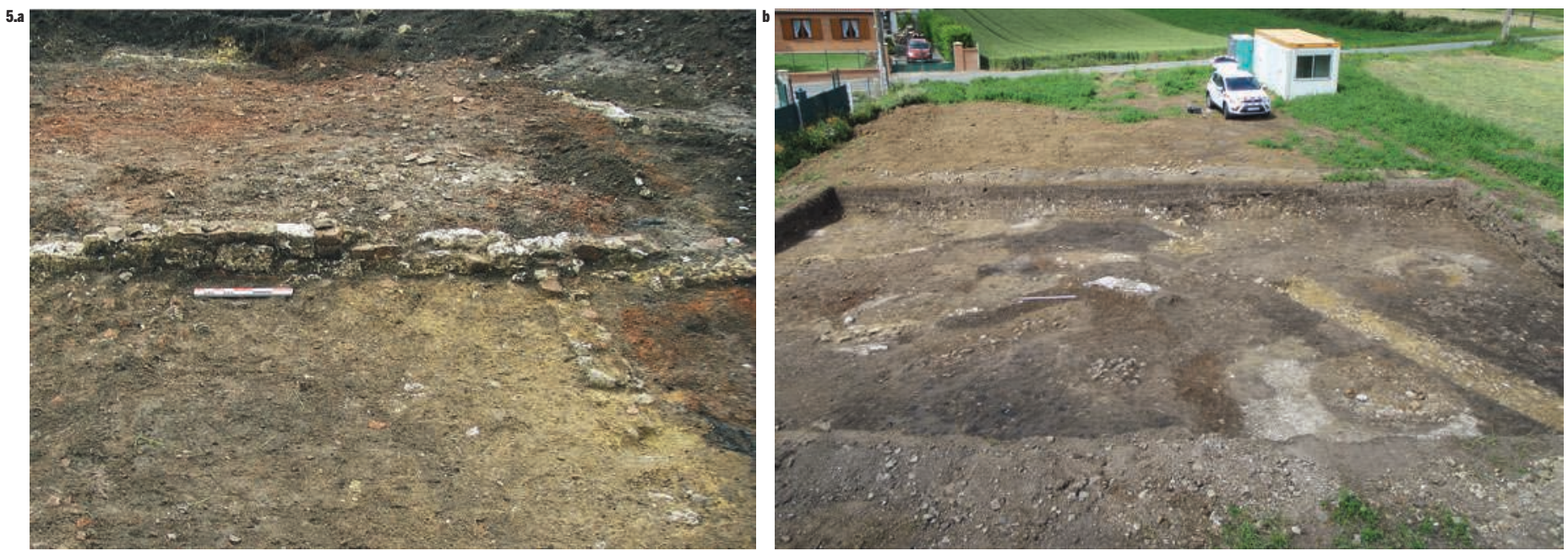
mobilier issu des remplissages, peu abondant, est daté de la fin du Moyen Âge ou du début de l'époque moderne mais ne peut être rattaché avec certitude à la campagne militaire de 1553.

La décision de Charles Quint de raser la ville eut, à l'époque, un retentissement considérable. De nombreuses poésies ou complaintes retracent le passé glorieux de la cité et perpétuent le souvenir de sa destruction (voir par exemple Laplane, 1856 ; Pigault de Beaupré, 1856 ; Quenson, 1855 et 1856). Mais quelles étaient les motivations de l'empereur? Sans doute voulait-il laver l'affront de ses troupes qui avaient connu, quelques mois plus tôt, un échec retentissant dans leur tentative de reprendre la ville de Metz aux Français. Par ailleurs, les dégâts causés par les deux mois de siège et le bombardement incessant de la ville et de ses murailles étaient considérables, comme en témoignent les récits des contemporains, les quelques gravures représentant le siège [ill. 2 et 3] ou les fouilles archéologiques récentes. Nombreux sont en effet les sondages qui livrent des traces d'incendie du dernier état de l'habitat civil (par exemple Blamangin, Vincent, 2011). Mais tout aussi nombreuses sont les interventions archéologiques où les indices d'une destruction violente par le feu sont absents, ou localisés, qui démontrent que celle-ci ne fut pas systématique (par exemple Barret et al., 2005 ou Labarre, 2012). Données archéologiques et sources écrites sont ici concordantes : aussi dévastateurs que furent le siège de la ville et le sac qui suivit, la cité aurait pu se relever - elle l'avait fait précédemment - si la décision politique de la raser n'avait pas été prise. Certes, le coût d'une remise en état des fortifications, pour assurer durablement le maintien de la place dans le giron impérial, aurait sans doute été considérable, alors que la topographie du site, dominé par de nombreuses collines, était une faiblesse majeure face aux progrès de l'artillerie (Martens, 2007). Surtout, Thérouanne, en retrait de la frontière, n'était pas d'un grand intérêt pour la défense des États d'Artois alors que le risque d'une reprise de la ville par les troupes d'Henri II pouvait, à tout moment, rouvrir «la mauvaise fenestre dont le vent françois se desgorge » (Jean Molinet [1435-1507] éd. Buchon, 1827-1828, p. 20o) sur les terres des anciens Pays-Bas espagnols. Finalement, comme le suggère Pieter Martens, la destruction totale de Thérouanne et d'Hesdin, et donc la suppression de ces enclaves, participaient avant tout d'une rationalisation et d'une tentative de stabilisation de la frontière sud-ouest de l'Artois.

Avant sa destruction, Thérouanne possédait, pour reprendre les termes d'un érudit du XIX ${ }^{\mathrm{e}}$ siècle, « la plus vaste, la plus haute et la plus belle cathédrale de la région du Nord » (Bled, 1895, p. 3). Les édifices religieux de la cité épiscopale [ill. 4] et les maisons des chanoines avaient été préservés des destructions, pourtant importantes, qui suivirent la prise de la ville en 1513 (Guyotjeannin, 2010). Le sort qui leur fut réservé en 1553 illustre bien le caractère exceptionnel de la décision impériale.
Aucun des lieux de culte n'échappa au sac de la ville. Charles Quint reconnut cependant que le pillage des objets du culte n'était pas conforme aux usages « de bonne et ancienne guerre » et exigea que quiconque possédait «corps saints, reliquiaires, vaisseaulx d'or ou d'argent, calices, croix, tapisseries, livres, registres, letraiges, cartulaires, comptes ou autres meubles... » les ramena aux chanoines de la cathédrale réfugiés à Saint-Omer², qu'ils aient été pris à l'occasion du sac ou rachetés aux soldats (Arch. de la ville de Lille, citée par La Fons, 1861). Pour autant, les ordres de Charles Quint étaient sans ambiguïtés : « On ne détruira pas seulement les édifices profanes mais encore les églises, les monastères et les hôpitaux » (Legrand, 1857-1861, p. 782), ce qui fut fait, aussi bien pour la cathédrale que pour les églises paroissiales. L'empereur souhaitait supprimer définitivement une enclave française sur ses terres, mais aussi démanteler un puissant diocèse, dont l'évêque nommé par le roi de France disposait d'une juridiction spirituelle qui s'étendait de part et d'autre de la frontière [ill. 1].

La destruction méthodique d'une ville comme Thérouanne est, à cette échelle, un processus complexe, souvent difficile à appréhender, qui nécessite des moyens humains et matériels considérables, une organisation des circulations, de la récupération des matériaux, de leur stockage et de leur transport, etc. Une abondante correspondance, notamment entre Marie de Hongrie, sœur de Charles Quint, régente des PaysBas, et Jean de Morbecque, capitaine de la ville voisine d'Aire-sur-1a-Lys, chargé de la destruction, éclaire l'avancement de l'entreprise, achevée avant la fin août 1553 (Martens, 2007 ; voir également la correspondance de ces derniers avec l'échevinage de Saint-Omer de Lille : Legrand, 1857-1861; La Fons, 1861). L'empereur somma les États de Flandre et d'Artois de mettre à disposition les pionniers nécessaires, y compris par la contrainte, et d'assurer le financement des travaux. Si les huit mille hommes promis par les provinces ne purent être réunis, les rôles de réquisition témoignent de la mobilisation : les villes d'Arras et Saint-Omer fournirent chacune 400 ouvriers, Lens 300, SaintPol et Béthune 200, Aire-sur-la-Lys 7o, etc. (Bled, 1895). Le nombre de pionniers étant insuffisant, tous les sujets de l'empereur furent autorisés à «prendre et enmener [les matériaux issus de la démolition] tant qu'il en vouldra », si bien qu'à la mi-juillet, Jean de Morbecque se déclarait incapable d'estimer le nombre de chariots qui avaient ainsi quitté la ville (lettre de Jean de Morbecque à Marie de Hongrie, 15 juillet 1553, Martens, 2007, p. 113). On fit sauter à la poudre les tours de l'enceinte et l'on procéda par sape et incendie des étançons pour provoquer l'effondrement des courtines dans le fossé (Martens, 2007). Le reste des terres du rempart, « exescivement hault » et "fort large », était « getté dedens le fossé » (lettre de Jean de Morbecque à Marie de Hongrie,
2. Les chanoines de

'ils étaie de France, à Boulogne-

sur-Mer après avoir été faits prisonniers et libérés contre rançon, et

s'ils étaient sujets du ro d’Espagne à Saint-Omer ou à Ypres (Bled, 1898). 


\begin{tabular}{|c|c|}
\hline $\begin{array}{l}\text { 1.Sur cette photographie } \\
\text { aérienne de la us vieille } \\
\text { ville in de Thérouanne prise } \\
\text { en } 1976 \text { par R.Agache, on } \\
\text { observe des traces de la cité, } \\
\text { en particulier certaines } \\
\text { rues et l'emplacement } \\
\text { de l'enceinte. } \\
\text { 2. Les observations } \\
\text { archéologiques, } \\
\text { confrontées aux plans } \\
\text { et vues anciennes, aux } \\
\text { éléments fossilisés dans } \\
\text { le parcellaire et le paysage, } \\
\text { aux photographies des } \\
\text { prospections aériennes } \\
\text { ou aux orthophotographies } \\
\text { contemporaines, } \\
\text { permettent de proposer } \\
\text { une restitution de la } \\
\text { topographie urbaine de } \\
\text { Thérouanne à la veille du } \\
\text { siège de 1553. }\end{array}$ & $\begin{array}{l}\text { Interventions } \\
\text { archéologiques citées dans } \\
\text { cet article: } \\
\text { 1.Fouilles de la cathédrale } \\
\text { par Camille Enlart,1898- } \\
\text { 1910; Roland Delmaire et } \\
\text { Honoré Bernard,1961-1980. } \\
\text { 2. Fouille du quartier } \\
\text { canonial,1981-1988 et } \\
\text { diagnostic «Vieille ville ॥, } \\
2000 \text { (Bernard,1985 } \\
\text { et Hosdez,2000). } \\
\text { 3.Fouille du contoumement } \\
\text { Nord-Ouest,1995 } \\
\text { (Blamangin,1996). } \\
\text { 4.Sondages programmés } \\
\text { de l'École des Chartes,1997- } \\
\text { 2002 (Barret et al.,2005). } \\
\text { 5. Diagnostic rue Saint-Jean, } \\
\text { parcelle AB 249 } \\
\text { (Blamangin,Vincent, 2011). } \\
\text { 6. Diagnostic Jardin } \\
\text { archéologique,2010 } \\
\text { (Maniez, 2010). } \\
\text { 7.Diagnostic rue Saint Jean, } \\
\text { parcelle AB 232 } \\
\text { (Labarre,2012). }\end{array}$ \\
\hline
\end{tabular}

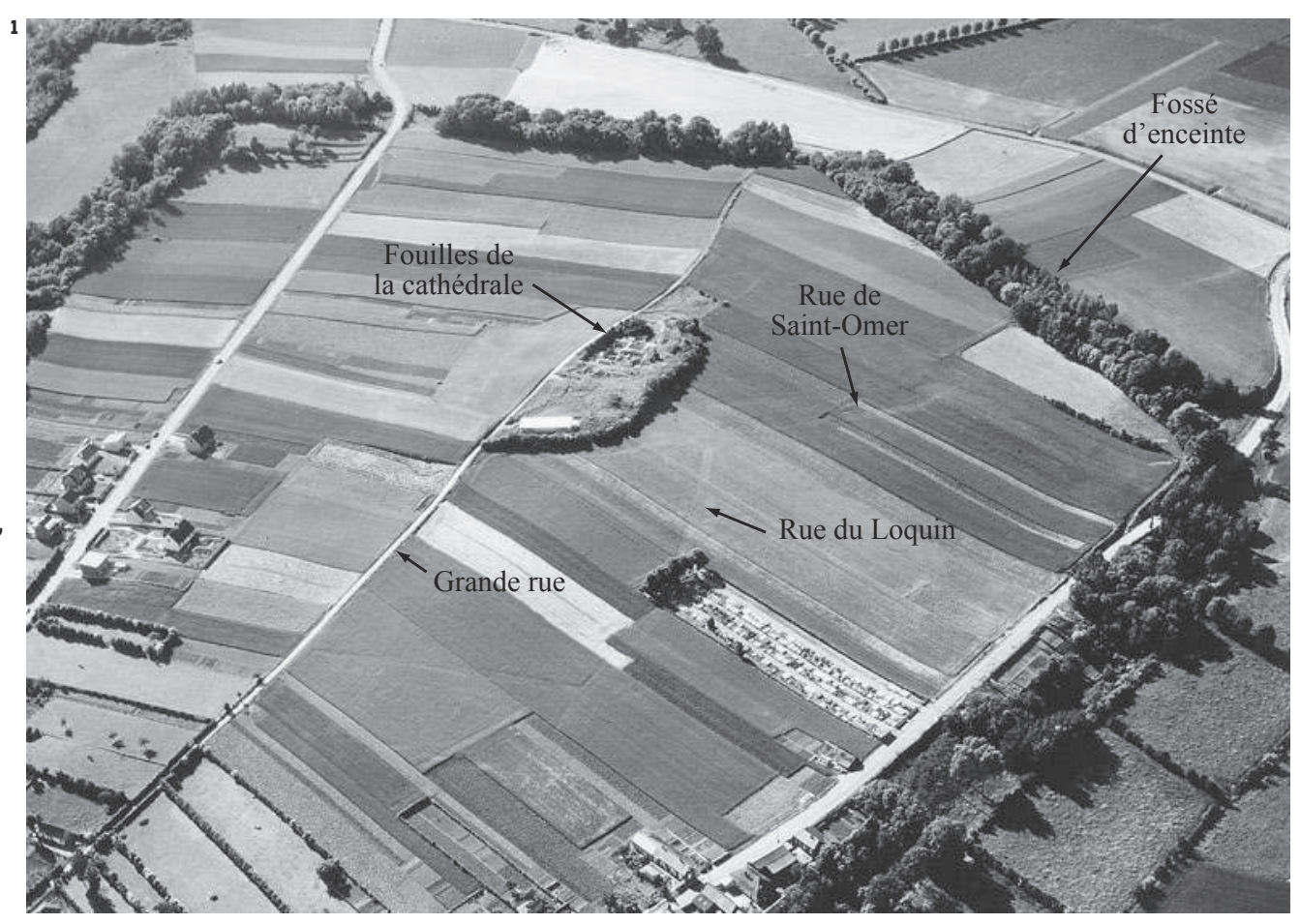

2

2

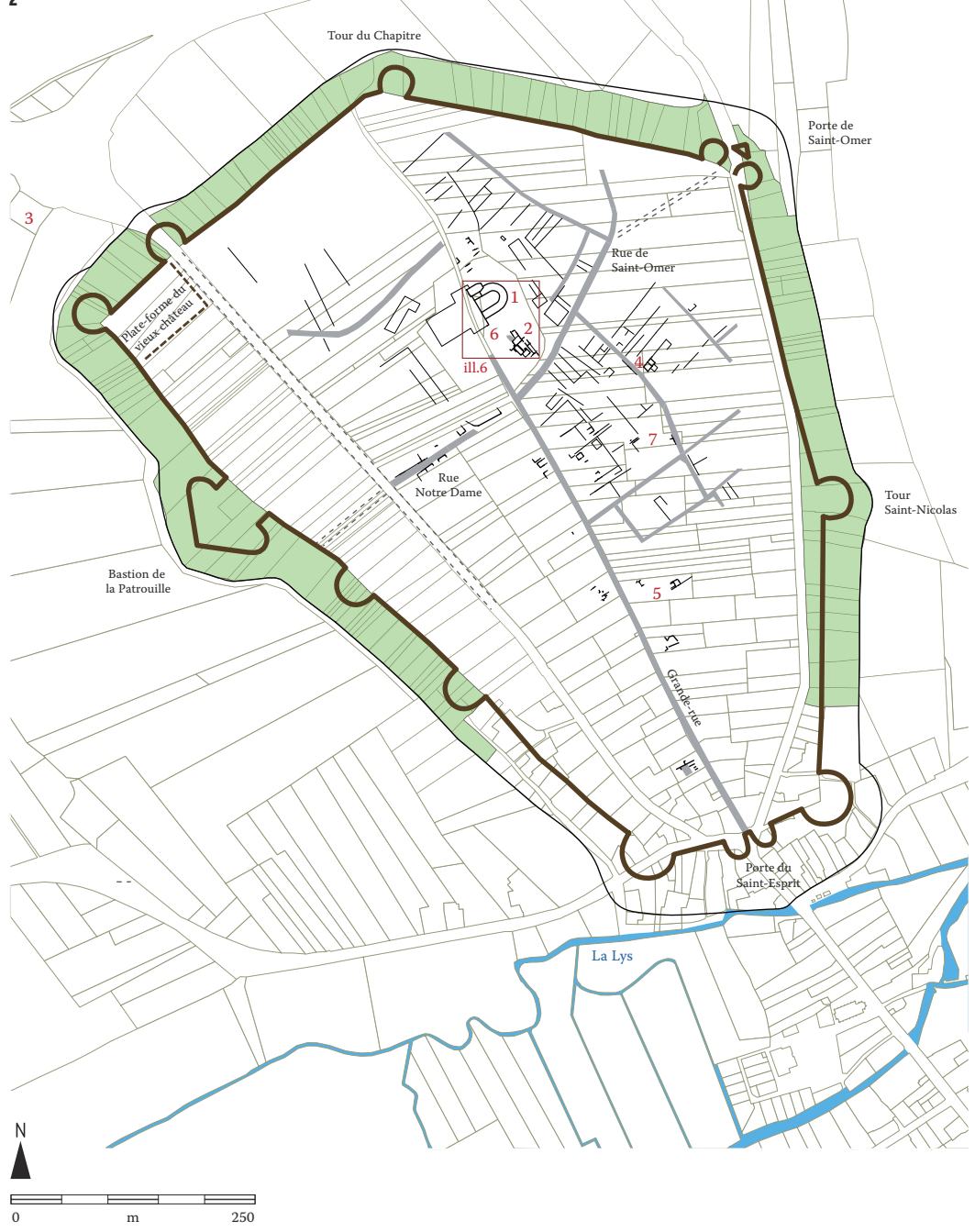

- Restitution du tracé de l'enceinte

Rideau d'arbres marquant l'emprise de l'ancien fossé

-: : Réseau viaire

— Fondations / parcellaire
Topographie urbaine de Thérouanne à là veille du siège de 1553

Une soixantaine d'opérations archéologiques (principalement des diagnostics sur des projets de construction de pavillon, mais aussi quelques fouilles préventives et sondages programmés) ont été réalisées sur le territoire de la commune de Thérouanne depuis le depuis des années 1990. Trente-huit sont localisées au sein du périmètre de la « Vieille ville ", détruite en 1553.

Les surfaces concernées, généralement restreintes, ne permettent pas d'appréhender l'organisation et l'évolution d'un quartier ou d'un îlot de la ville médiévale dans son ensemble. Pour autant, confrontés aux plans et vues anciennes, ainsi qu'aux photos aériennes des prospections de Roger Agache réalisées en 1976 [ill. 1] ou aux orthophotographies contemporaines, les résultats de ces opérations archéologiques dessinent progressivement la trame urbaine, telle qu'elle était à la veille du démantèlement de la cité [ill. 2], avec son réseau viaire, ses parcelles allongées avec habitations en pignon de rue, une spécialisation des quartiers, etc. La cathédrale, au centre, dominait la ville. Le palais épiscopal était installé plus à l'ouest et au nord de la rue NotreDame, tandis que le quartier canonial se développait à l'est de la cathédrale, au nord de la rue de SaintOmer. L'ancien château, au nord-ouest, avait été arasé et transformé en terrasse d'artillerie. Fn 1553, deux portes permettaient l'entrée dans la ville : celle de Saint-Omer, à l'est, et celle du SaintFsprit, au sud. Depuis cette dernière, on accédait à la cathédrale par la Grande rue, dont l'actuelle rue Saint-Jean reprend. le tracé. Cet axe majeur de la cité médiévale est le seul chemin figuré, au milieu de la ville ruinée, sur le plan levé par le cartographe Jacques de Deventer peu après 1553 (copie du plan des vestiges de Thérouanne par Jacques de Deventer, AD Pas-de-Calais, 4 J-439-1 16). Il joua sans doute un rôle majeur dans les opérations de démolition et pour l'évacuation des charrois de matériaux récupérés. 
23 juillet 1553, Martens, 2007, p. 114), qui fut ainsi partiellement remblayé. Son tracé est encore fossilisé dans le parcellaire et bien visible dans le paysage, marqué sur l'essentiel du pourtour par une profonde dépression du terrain et un rideau végétal [cf. encadrép 68]. Les sources textuelles rendent ainsi compte de la priorité donnée au démantèlement des fortifications, afin que les Français ne puissent reprendre et défendre la place, mais nous renseignent finalement bien peu sur la chronologie et les moyens consacrés à la destruction des édifices civils et religieux. Le 15 août 1553, alors que la démolition de l'enceinte était pratiquement achevée et que les dernières troupes impériales s'apprêtaient à quitter la ville, la cathédrale était encore, au moins partiellement, en élévation : ordre fut donner de «faire tomber ou brusler le comble de l'église » (lettre d'Emmanuel-Philibert de Savoie à Jean de Morbecque, 15 août 1553, Martens, 2007, p. 117) avant que les soldats ne se retirent.

Les interventions archéologiques confirment le caractère systématique de la démolition : les ouvriers ne se contentèrent pas de provoquer l'effondrement des édifices mais toutes les élévations, de terre comme de brique ou de pierre, furent soigneusement arasées. Il n'en subsiste en général qu'une ou deux assises [ill. 5a et b]. Les caves et les puits furent remblayés (notamment Bernard, 1985 ; Hosdez, 2000). Une couche de gravats, composée de fragments de terres cuites architecturales (briques, tuiles, parfois carreaux de pavement), de petits moellons calcaire, de rares blocs taillés et de nodules de mortier de chaux ou, dans les secteurs d'habitats plus modestes, de torchis, couvre la presque totalité des 36 hectares du site. Généralement épais de quelques dizaines de centimètres, ce niveau de démolition peut atteindre, par endroit, plusieurs mètres. On s'attaqua parfois «aux fondemens » des édifices les plus importants pour en réutiliser les parements, au point qu'il ne subsiste souvent que le blocage interne de leurs fondations, comme pour le chœur de la cathédrale. Le pavement de certaines voiries fut soigneusement récupéré (Labarre, 2012; Barret et al., 2005) et la ville dans son ensemble servit de carrière de pierres, utilisées notamment pour le renforcement des remparts de Saint-Omer. Les ruines de la cité ont approvisionné en matériaux, pendant de longues années encore, les chantiers de la région, comme en témoignent les nombreuses fosses de spoliation qui viennent percer les niveaux de gravats. En juin 1559, alors que Thérouanne était rendue à la maison de Valois, l'ambassadeur de France auprès du roi d'Espagne se plaignait encore de la présence sur le site, chaque jour, de centaines de sujets des États d'Artois venus « prendre et remporter le reste des démolitions » (Bled, 1895, p. 18). Lorsqu'il vint lever les plans de la ville détruite en 1730, l'ingénieur Claude-Félix Masse notait: «les habitans en ont enlevez tous les matériaux, si bien que [...] je n'y remarquai que deux pierres de moyenne grosseur, et tout le reste des débris n'étant

que de mauvais moellon qui n'a point vallu la peine d'être charrié ». Son plan figure, sur les terres de l'ancienne cité, le chaos des «buttes de terres entrecoupés en une infinité d'endroits par les trous qu'ont faits les carellieurs » (Claude-Félix Masse, Plan des vestiges de l'ancienne ville de Thérouanne et mémoire adjoint, en date des 15 et 30 octobre 1730 , fol. 3 et 6v, Archives du Génie, Service historique des armés, Vincennes, cité et reproduit par Martens, 2007).

Un diagnostic archéologique récent, réalisé par le Centre départemental d'archéologie du Pas-deCalais (Maniez, 2010) ${ }^{\mathbf{3}}$, apporte des informations inédites sur la cathédrale disparue et démontre l'intérêt de traiter ces remblais de démolition comme de véritables niveaux archéologiques. L'intervention portait sur le quartier canonial entourant le chœur de la cathédrale médiévale [ill.6]. Elle devait documenter les cotes d'apparition et l'état de conservation des vestiges antiques et/ou médiévaux, mais aussi mettre en évidence les zones n'ayant subi aucune investigation archéologique dans un secteur partiellement fouillé entre 1898 et 1910, puis de 1961 à la fin des années 1980. Il avait été décidé de ne pas sonder l'emprise de la cathédrale elle-même, dont les vestiges apparents du chœur, très altérés par le temps, avaient été ré-enfouis en 2009.

Les plans et vues de la ville postérieurs à sa destruction montrent que les parcelles occupées par les ruines de l'ancienne cathédrale n'ont jamais été remises en culture, ce qui explique sans doute le parfait état de conservation du niveau de démolition de l'édifice religieux. Le niveau de gravats encore intact, hormis sur la zone anciennement fouillée, apparaît immédiatement sous la « terre végétale » [ill. 7]. Il atteint $2 \mathrm{~m}$ d'épaisseur au plus près du transept sud de l'édifice religieux et comporte de très nombreux éléments architecturaux et décoratifs, issus du registre de l'iconographie religieuse [ill. 8]. Contrairement à la plus grande part des blocs lapidaires découverts sur le territoire de la commune, qui sont généralement isolés de tout contexte, les 61 blocs en calcaire fin et de couleur beige mis au jour à l'occasion de ce diagnostic proviennent d'un même niveau de démolition non remanié. Certains éléments présentent des traces de polychromie (pigments rouges, bleus, brun-roux) appliquée sur un enduit blanc ou des traces de dorure. Ils peuvent être regroupés selon les différents registres de l'ornementation d'un portail, du bas relief jusqu'aux scènes décoratives ornant les voussures de l'archivolte. De nombreuses analogies, notamment les dais avec coussin sur lesquels reposent les grandes statues de l'ébrasement, peuvent être établies avec l'iconographie du portail sud du transept de la cathédrale Notre-Dame d'Amiens. L'étude confirme ainsi que cet ensemble exceptionnel est issu du décor ornemental du portail méridional de la cathédrale de Thérouanne, typique des styles gothiques classique et rayonnant de la fin du XIII ${ }^{\mathrm{e}}$ siècle [ill. 9 et encadré p 30].

Le niveau de gravats constituant un terminus ad quem étant conservé, il est fort probable que, dans du «Jardin archéologique

de Thérouanne, rue

Saint-Jean, réalisé

en octobre 2010 sous

la direction de Jérôme

Maniez. 


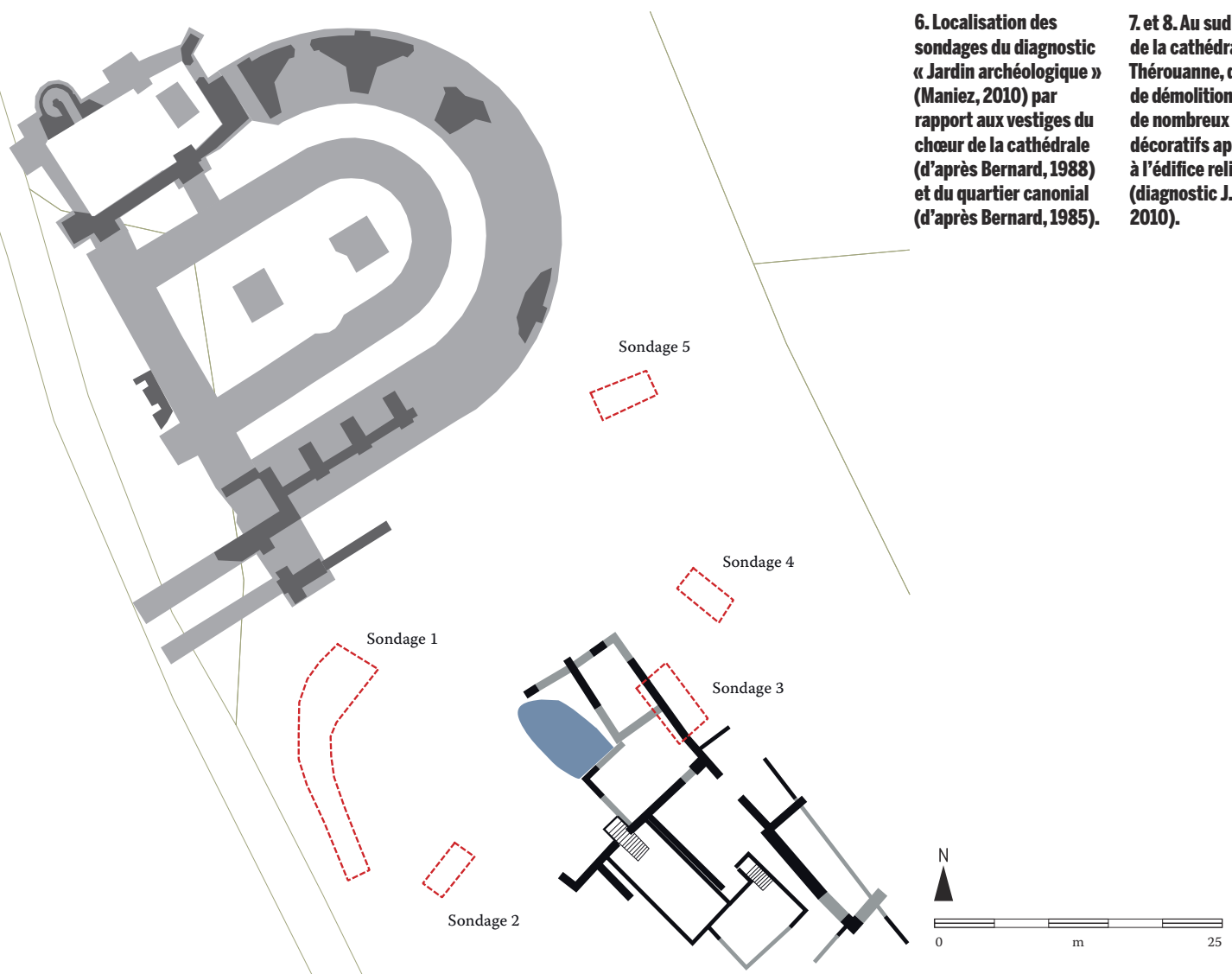

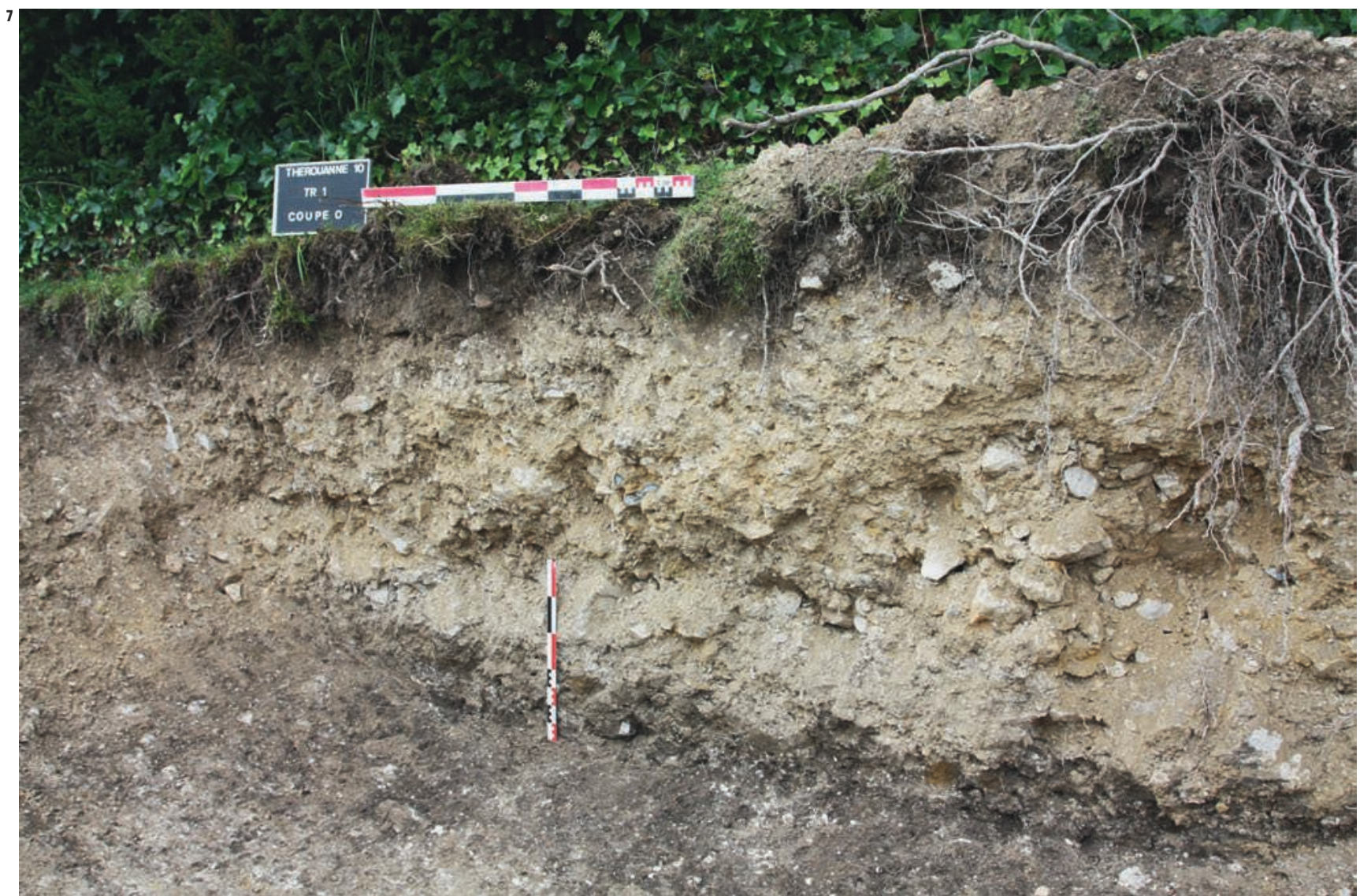




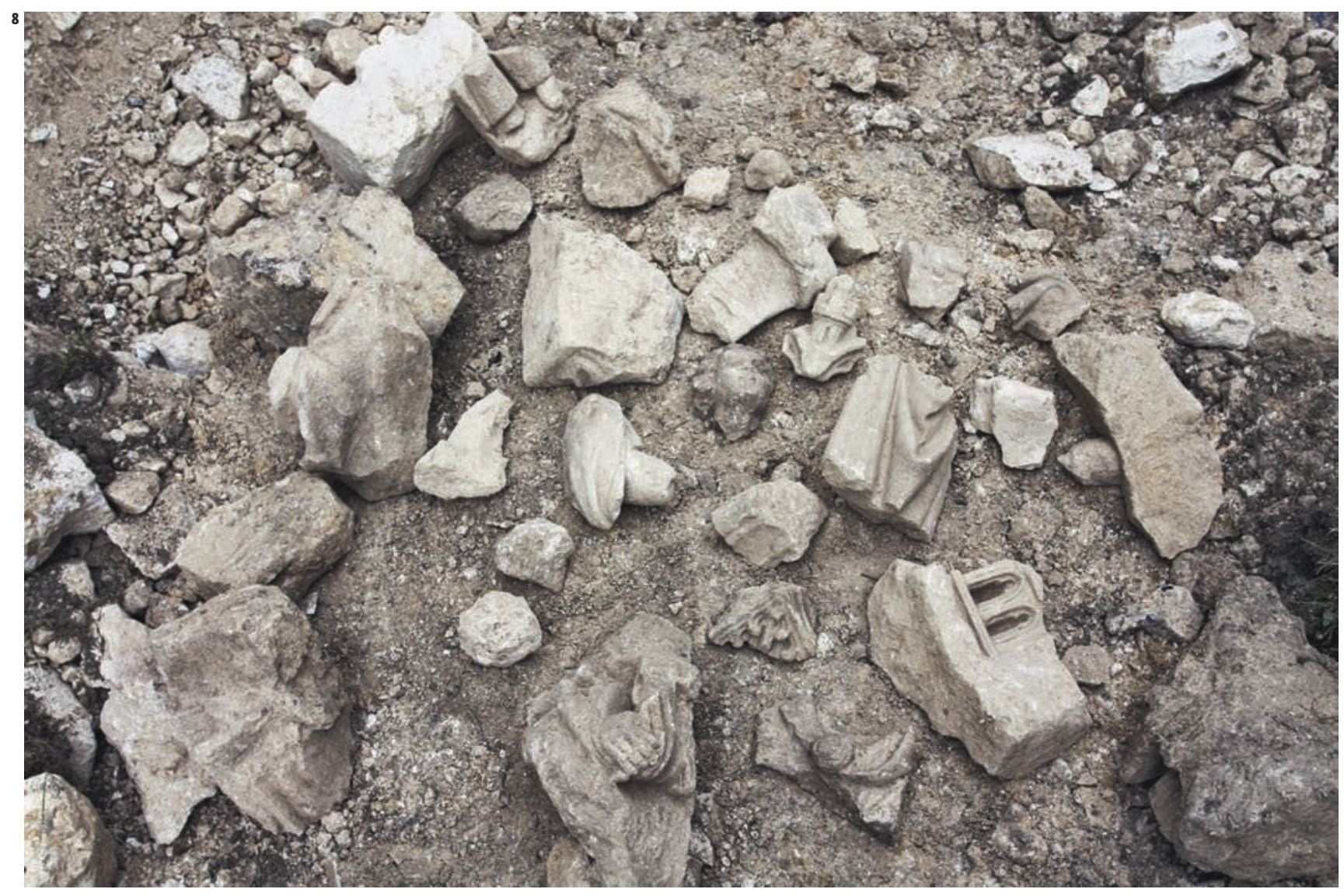

(2) $x$ fortate -

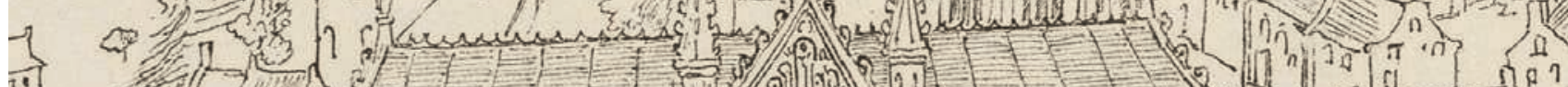
iin 3 a

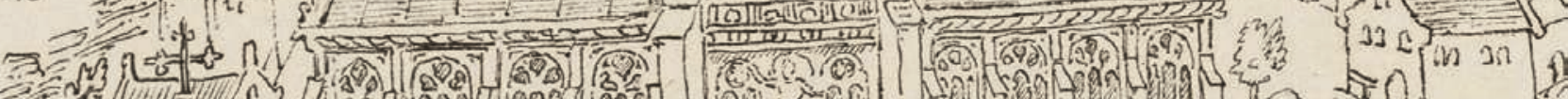
i.

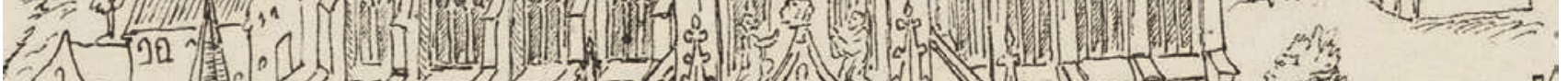
3.

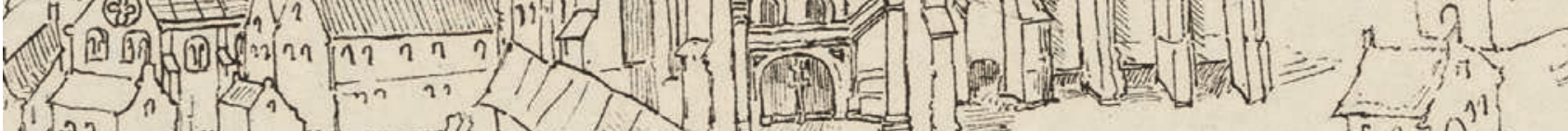

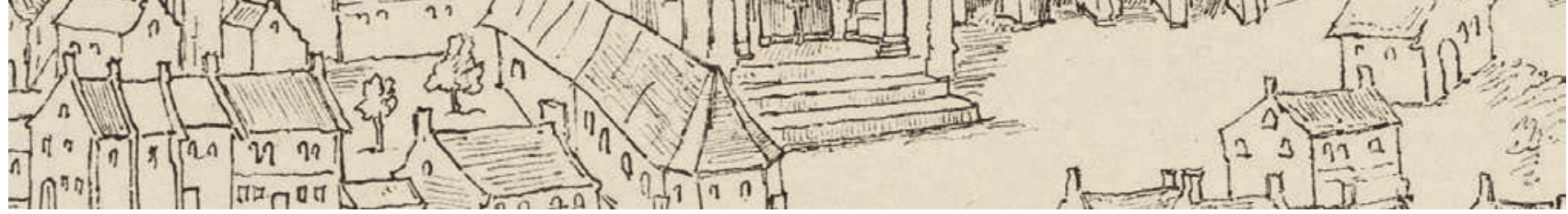




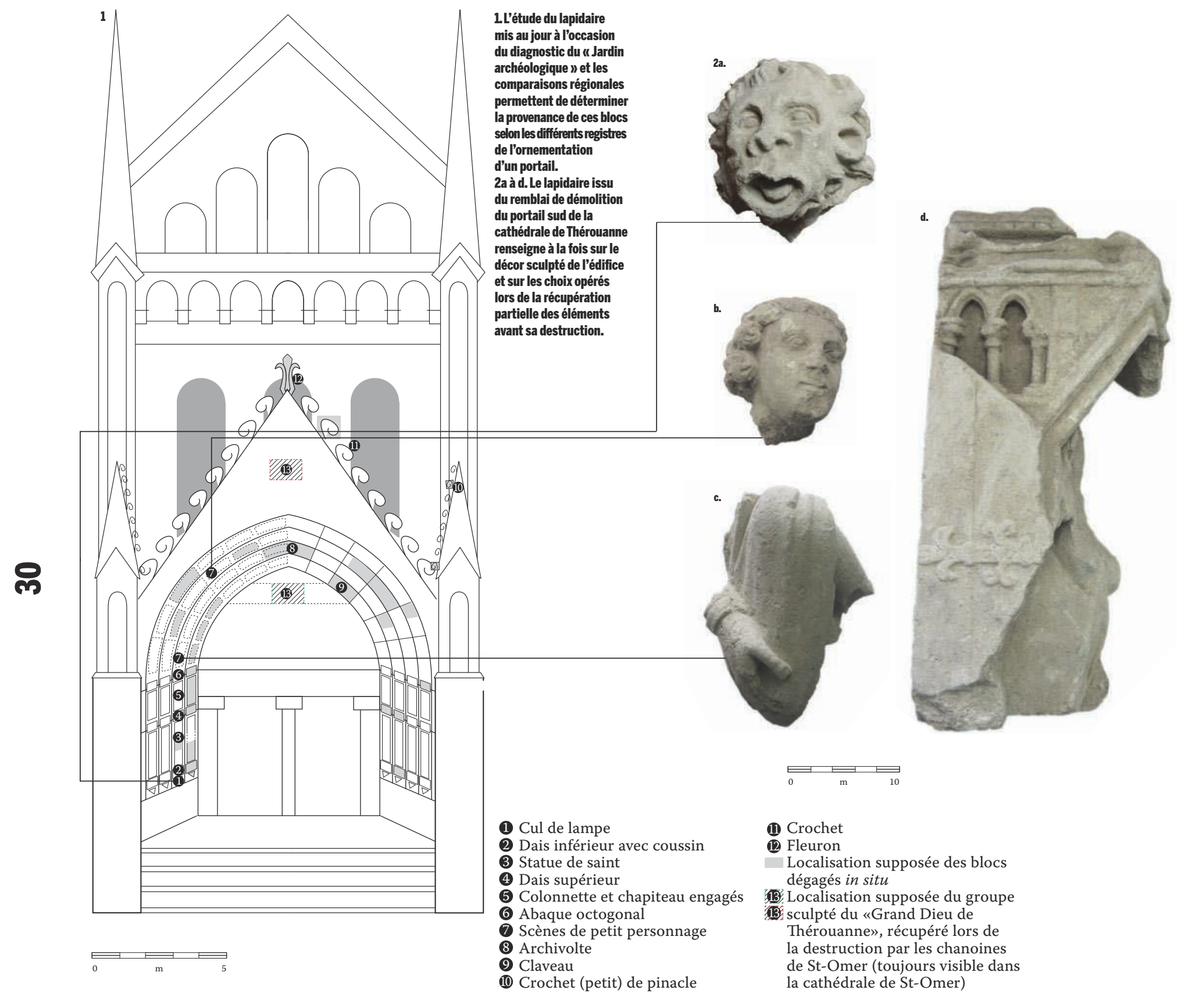

\section{Démolition et récupération du portail sud de la cathédrale de Thérouanne}

Au lendemain de la prise de la ville par les troupes impériales, le portail de la cathédrale de Thérouanne fut donné aux chanoines de Saint-Omer pour orner leur collégiale nouvellement achevée. Les registres capitulaires et les comptes de la fabrique détaillent les péripéties et les dépenses engagées pour cette entreprise de récupération (Deschamps de Pas, 1852), achevée dans les premières semaines de septembre 1553 : envoi d'un dessinateur à Thérouanne pour réaliser un patron du portail avant qu'une mission chargée d'organiser son démontage et le transport ne se rende compte qu'il n'était pas adapté à la nouvelle église de SaintOmer ; décision prise par le chapitre de ne prendre que les plus belles sculptures; achat de chevaux, mobilisation des échafaudages, cordages et engins nécessaires ; recrutement des mancuvres, interventions du charpentier et du maître maçon, réquisition des fermiers dépendants de la collégiale pour assurer le transport ; transport des figures du Jugement, de Moïse et du Salvator et de quelques autres images, etc.

Le groupe du. Jugement, plus connu sous le nom de « Grand Dieu de Thérouanne », est encore aujourd'hui conservé dans la cathédrale de SaintOmer. I'ensemble de blocs issus du diagnostic de 2010 confirme à la fois l'intensité de cette récupération et son caractère sélectif, avec le délaissement de l'essentiel du décor sculpté, à l'exception des grandes « figures » [ill. 1]. En effet, les blocs architecturaux, tels que les fragments de voussures, de claveau ou de tailloir, plus facilement réutilisables pour des constructions civiles, sont faiblement représentés. À l'inverse, avec 49 blocs finement sculptés sur les 61 mis au jour, les éléments purement figuratifs qui ne peuvent être réemployés, comme les têtes, les drapés, les dais et les mains, dominent nettement le corpus [ill. 2a à d] . 
ce secteur, les niveaux d'occupation antérieurs à la destruction de la ville le soient également, ce qui a pu être observé dans la partie nord du site, où ils apparaissent directement sous les horizons organiques. Ils se composent de niveaux de sol, de niveaux de circulation en craie damée, de murs (fondations et première assise d'élévations) ainsi que d'une probable sépulture. Uniquement dégagés en plan dans le cadre du diagnostic, ces différents éléments appartiennent aux bâtiments du quartier canonial entourant la cathédrale et complètent les informations recueillies par Honoré Bernard sur ce secteur de la ville (Bernard, 1985). Ils correspondent au dernier état de l'occupation du site, avant destruction. Les observations de terrain montrent toutefois la présence de vestiges antérieurs (reprises de murs, présence de mortier de tuileau antique, etc.). Le potentiel archéologique du site est préservé.

En avril 1559, le traité du Cateau-Cambrésis mettait fin au conflit entre Henri II et Philippe II d'Espagne et réglait définitivement le sort de Thérouanne. La ville, ou plutôt ses ruines, furent restituées à la couronne de France, avec interdiction faite à celle-ci de reconstruire une forteresse. Le même traité autorisait les Français à détruire, en représailles, la ville impériale ardennaise d'Ivoys
(Carignan). Quelques semaines plus tard, le pape Paul IV entérinait la partition du diocèse des Morins, dont les territoires septentrionaux viendront former les nouveaux diocèses d'Ypres et de Saint-Omer, érigés dès 1560, tandis que le siège épiscopal de la partie française est déplacé à Boulogne-sur-Mer en 1567. Les possessions et rentes de l'ancien évêché furent divisées entre les parties françaises et espagnoles. Le fief, et donc les terrains de la ville détruite, restèrent cependant, jusqu'à la Révolution française, possession commune des évêques de Boulogne, Saint-Omer et Ypres. Ils les louaient comme pâturage aux habitants du nouveau village de Thérouanne, implanté plus au sud, sur les terrains de l'ancien faubourg dit du Saint-Esprit. Cette possession commune, qui interdisait toute aliénation du fond (Bled, 1895), permit qu'aucune construction ne vint s'installer sur les terrains de l'ancienne ville jusqu'à la fin du XVIII ${ }^{\mathrm{e}}$ siècle. Protégés des reconstructions, les vestiges de la ville, dans leur dernier état avant démantèlement, sont ainsi remarquablement conservés. Ils forment un ensemble d'une rare cohérence et, de fait, une extraordinaire réserve archéologique.

\section{Références bibliographiques}

Buchon J.-A., 1827-1828, Chroniques de Jean Molinet, publiées pour la première fois d'après les manuscrits de la Bibliothèque du Roi, t. 2 , Collection des chroniques nationales françaises, t. XLIV, Paris, Verdière, $467 \mathrm{p}$.

LA Fons A. DE, 1861, « Documents inédits sur la prise de Thérouanne (1553) », Bulletin historique de la Société des antiquaires de la Morinie, vol. II, $\mathrm{n}^{\circ}$ 27-28, p. 596-60o.

LAPLANE H. DE, 1856, « Autre complainte de Thérouane », Bulletin historique de la Société des antiquaires de la Morinie, vol. II, n 17-18, p. 146-154.

LEGRAND A., 1857-1861, « Correspondance inédite des généraux de l'armée de l'Empereur Charles-Quint avec les Mayeurs et Echevins de la ville de St-Omer, à l'occasion du siège, prise et destruction de la ville de Thérouanne en 1553 ", Bulletin historique de la Société des antiquaires de la Morinie, vol. II, $\mathrm{n}^{\circ}$ 31-36, p. 720-737, 778-79o et 932-940.

Pigault De Beaupré A., 1856, « Chanson ancienne sur la destruction de la ville de Thérouanne en 1553 ", Bulletin de la Société des antiquaires de la Morinie, vol. $2, \mathrm{n}^{\circ} 14-15$, p. 43-48.

QUenson F., 1855, "Complainte de Thérouene », Bulletin de la Société des antiquaires de la Morinie, vol. $1, n^{\circ} 12$, p. 26o-266.

RABUtin F. DE, 1932, Commentaires des guerres en la Gaule belgique (1551-1559), t. 1 (1551-1555), publiés par Ch. Gailly de Taurines pour la Société de l'histoire de France, Paris, Êd. H. Champion, 346 p.

Barbé H., Thuillier F., 1993, « Thérouanne, la vieille ville - rue Saint-Jean, terrain Tétard », Bilan scientifique de la région Nord-Pas-de-Calais, p. 82-83.

Barret E., Gérardot A., Noyé G., 2005, Thérouanne, «La Vieille Ville», rapport préliminaire sur les sondages de l'École des Chartes, 1997-2002, Rapport de fouille programmée, SRA Nord-Pasde-Calais, 2 vol., 179 et 182 p.

BERnARD H., 1980, « Remarques et hypothèse sur le développement urbain de Thérouanne (Pas-deCalais) ", Septentrion, 10, p. 41-6o.

BERNARD H., 1985, « Thérouanne : les fouilles du quartier canonial », Archéologie médiévale, 15, p. 157-189.
BERNARD H., 1988, « Une restitution de l'ancienne cathédrale de Thérouanne ", Archéologie médiévale, 18, p. 141-177.

Blamangin O., 1996, « Thérouanne - déviation NordOuest, 'Le bois Robichet', Habitat péri-urbain et nécropole », in BARBÉ H. (dir.), Du terrain au musée... 1993-1996. Trois années de recherches archéologiques dans l'Audomarois, catalogue d'exposition, Saint-Omer, p. 54-61.

Blamangin O., Devred V., Leriche B., 2011, « L'apport de l'archéologie préventive à la connaissance de la topographie antique de la ville de Thérouanne ", Revue du Nord - Archéologie de la Picardie et du Nord de la France, t. 93, n 393, p. 37-77.

Blamangin O., VINCENT V., 2011, «Un atelier de potier médiéval au cœur de la ville fortifiée de Thérouanne », Revue du Nord - Archéologie de la Picardie et du Nord de la France, t. 93, n 393, p. 261-276.

BLED O., 1895, Thérouanne, une ville disparue, Paris, Imprimerie nationale, 28 p. et pl. (Extrait du Bulletin historique et philologique du Comité des travaux historiques et scientifiques, 1894).

BLED O., 1898, Les évêques de Saint-Omer depuis la chute de Thérouanne, t. 1,1553-1619, Saint-Omer, Imprimerie d'Homont (Mémoires de la Société des antiquaires de la Morinie, t. 26), 432 p. et 103 p.

Delmaire B., 1999, « Thérouanne et Hesdin : Deux destructions (1553), une reconstruction », in Destruction et reconstruction de villes, du Moyen Âge à nos jours. Actes du colloque international de Spa, 10-12 septembre 1996, Bruxelles, Crédit Communal, p. 127-153.

Delmaire B., 2010, «La géographie paroissiale du Diocèse de Thérouanne ( $\mathrm{XIV}^{\mathrm{e}}-\mathrm{XVI}^{\mathrm{e}}$ siècle) : approche cartographique ", in RIDER J. et Tоск B.-M. (éd.), Le diocèse de Thérouanne au Moyen Âge. Actes de la journée d'étude tenue à Lille le 3 mai 2007, Arras, Commission départementale d'histoire et d'archéologie du Pas-de-Calais (Mémoires, t. 39), p. 149-180.

Delmaire R., 1976, Étude archéologique de la partie orientale de la Cité des Morins : Civitas Morinorum, Arras, Commission départementale des monuments historiques du Pas-de-Calais (Mémoires, t. 16), 410 p.
Delmaire R., 1984, « Notes sur l'évolution urbaine de Thérouanne ", Revue archéologique de Picardie, $n^{\circ} 3-4$, p. 223-229.

DeschampS De PAS L., 1852, « Translation à SaintOmer du portail de la cathédrale de Thérouanne ", Bulletin historique de la Société des antiquaires de la Morinie, vol. I, nº 4, p. 117-126.

Guyotjeannin O., 2010, «Thérouanne au bas Moyen Âge », in Rider J. et Tock B.-M. (éd.), Le diocèse de Thérouanne au Moyen Âge. Actes de la journée détude tenue à Lille le 3 mai 2007, Arras, Commission départementale d'histoire et d'archéologie du Pas-de-Calais (Mémoires, t. 39), p. 181-19o.

Hosdez C., 200o, Thérouanne, «La Vieille Ville», Rapport final d'opération, Afan.

LABARRE D., 2012, Thérouanne, parcelle $A B$ 232, Rapport de diagnostic, Inrap-SRA Nord-Pas-de-Calais, $80 \mathrm{p}$.

MAniez J., 2010, Thérouanne (62) - Cathédrale, rue Saint-Jean, Jardin archéologique, Rapport de diagnostic, Centre départemental d'archéologie du Pas-de-Calais, 50 p.

MARTENS P., 2007, « La destruction de Thérouanne et d'Hesdin par Charles Quint en 1553 ", in BLIECK G., Contamine P., Corvisier C., Faucherre N., MESQui J. (éd.), La forteresse à l'épreuve du temps. Destruction, dissolution, dénaturation, $X I^{e}-X X^{e}$ siècle, Paris, Éditions du CTHS, p. 63-117.

MARTENS P., 2011, « La puissance de l'artillerie de Charles Quint au milieu du XVI ${ }^{\mathrm{e}}$ siècle: le siège de Thérouanne en 1553 ", in Prouteau N., de Crouy-Channel E., FAUCHÈre N. (dir.), Artillerie et fortification, 1200-1600, Rennes, Presses universitaires de Rennes, Collection Archéologie \& Culture, p. 119-142.

MÉRIAUX C., 200o, «Thérouanne et son diocèse jusqu'à la fin de l'époque carolingienne : les étapes de la christianisation d'après les sources écrites ", Bibliothèque de l'École des chartes, t. 158, 2, p. 377-406.

Potter D., 2008, Renaissance France at War: Armies, Culture an Society, c1480-1560, Woodbridge, The Boydell Press, $408 \mathrm{p}$.

Quenson F., 1856, « Thérouanne détruite (20 juin 1553) ", Bulletin de la Société des antiquaires de la Morinie, vol. 2, n 16, p. 101-105.

VISSIÈRE L., 2000, «L'éternel gambit: Thérouanne sur l'échiquier européen (1477-1559) », Bulletin de la Commission départementale d'histoire et d'archéologie du Pas-de-Calais, XVIII, p. 61-106. 